Itinéraires Itinéraires

Littérature, textes, cultures

2020-3 | 2021

Le rap, une poésie de performances

\title{
«Dans la rue et le bitume ne poussent que les fleurs du mal » : jouer et déjouer Charles Baudelaire et sa poésie dans le rap français
}

(Re)playing Charles Baudelaire's Poetry in French Rap Music

\section{Nina Rolland}

\section{CpenEdition}

Journals

Édition électronique

URL : https://journals.openedition.org/itineraires/9377

DOI : 10.4000/itineraires.9377

ISSN : 2427-920X

Éditeur

Pléiade

\section{Référence électronique}

Nina Rolland, « «Dans la rue et le bitume ne poussent que les fleurs du mal » : jouer et déjouer Charles Baudelaire et sa poésie dans le rap français », Itinéraires [En ligne], 2020-3 | 2021, mis en ligne le 09 décembre 2021, consulté le 12 décembre 2021. URL : http://journals.openedition.org/itineraires/9377 ; DOI : https://doi.org/10.4000/itineraires.9377

Ce document a été généré automatiquement le 12 décembre 2021.

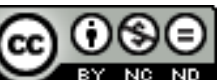

Itinéraires est mis à disposition selon les termes de la licence Creative Commons Attribution - Pas d'Utilisation Commerciale - Pas de Modification 4.0 International. 
« Dans la rue et le bitume ne poussent que les fleurs du mal »: jouer et déjouer Charles Baudelaire et sa poésie dans le rap français

\author{
(Re)playing Charles Baudelaire's Poetry in French Rap Music
}

Nina Rolland

\title{
Introduction
}

1 L'un des éléments constitutifs du rap est de réutiliser des matériaux sonores et textuels du passé et de les transformer. D'autres styles musicaux et formes artistiques empruntent également au passé mais la particularité du rap est de revendiquer la force créatrice de l'emprunt et de se positionner en culture «open source » (Williams 2013 : 1). Les samples de Chopin dans "That's my people» (1998) de NTM, de Schubert dans «Hip Hop forever » (2000) de Busta Flex, ou encore de Gabriel Fauré dans «Une place au premier rang» (2004) d'ATK, prouvent que les canons musical et littéraire du $\mathrm{XIX}^{\mathrm{e}}$ siècle sont une source d'inspiration pour les rappeurs et rappeuses. Parmi les poètes du siècle romantique, Charles Baudelaire est sans conteste la figure qui revient le plus souvent dans les textes de rap. Une comparaison des références aux patronymes de poètes dans les textes de rap sur la plateforme en ligne Genius.com nous montre que Baudelaire est mentionné dans 185 chansons de rap francophone, Rimbaud dans 63 chansons, et Victor Hugo dans 53 chansons ${ }^{1}$. Deux autres auteurs de siècles différents sont également régulièrement mentionnés: Aimé Césaire apparaît dans 94 chansons et Cyrano de Bergerac dans 31 chansons. Cependant, la figure littéraire la plus citée est celle de Molière; son nom apparaît dans 319 textes, bien que la vaste majorité des références concerne le syntagme « la langue de Molière ». Le nombre élevé de références à Baudelaire n'est, quant à lui, pas surprenant puisque le poète a inspiré 
les musiciens depuis la fin du XIX $x^{e}$ siècle jusqu'à aujourd'hui dans différents styles de musique.

Le Baudelaire Song Project ${ }^{2}$, a recensé plus de 1700 mises en musique de la poésie de Baudelaire dans vingt langues différentes et plus de quarante styles musicaux (de la musique classique à la pop en passant par le death metal) ${ }^{3}$. Le repérage des données s'est effectué en consultant des catalogues et archives (BNF, Centre international de la mélodie française), des bases de données (WorldCat, Genius, Discogs), des plateformes de streaming (Spotify, YouTube, SoundCloud) et à travers les réseaux sociaux ${ }^{4}$. Au-delà des célèbres mélodies de Duparc, Fauré et Debussy, le projet a mis en lumière des mises en musique moins connues, telles que les mélodies de compositrices ${ }^{5}$ ou des projets collaboratifs (Abbott 2019). Tous les poèmes des Fleurs du Mal, ainsi que treize poèmes en prose, ont été mis en musique ${ }^{6}$. La popularité de Charles Baudelaire auprès des musiciens peut s'expliquer par la forme musicale de certains de ses poèmes (comme «L'Invitation au voyage » qui comprend déjà un refrain ou le pantoum « Harmonie du soir » qui répète et entrelace certains vers), par la forme courte d'autres poèmes (notamment le sonnet), par l'universalité des thèmes abordés (l'amour, la mort, la mélancolie, etc.), mais aussi par la figure même du poète, à la fois poète maudit et moderne, canonique et non-conformiste. Pour Antoine Compagnon, Baudelaire est même moderne et antimoderne à la fois, il y a chez le poète "une ambivalence essentielle », une " dualité », une « réversibilité » (Compagnon 2014 : 7).

3 Certaines adaptations de la poésie de Baudelaire sont trop éloignées du texte original pour être recensées comme mises en musique. C'est le cas de nombreux morceaux de rap qui citent, samplent ou paraphrasent des vers de Baudelaire. Comme le souligne Bettina Ghio, il s'agit donc plus d'intertextualité que de mise en musique de poèmes (2016 : 49). L'intertextualité littéraire a été définie par Julia Kristeva d'après la théorie de Mikhail Bakhtine sur le dialogisme et la polyphonie : « tout texte se construit comme mosaïque de citations, tout texte est absorption et transformation d'un autre texte " (Kristeva 1969: 85). Le concept a ensuite été développé par de nombreux théoriciens qui ont différencié plusieurs degrés d'intertextualité. Dans Palimpsestes (1982), Gérard Genette identifie par exemple cinq types de "transtextualité » qui se recoupent, dont l'hypertextualité qui correspond à toute transformation d'un texte par un autre. Tiphaine Samoyault présente quant à elle l'intertextualité comme un espace de mémoire instable où le lecteur peut passer à côté des références (2001). Enfin, Paul Zumthor parle d'« intervocalité » pour souligner l'oralité des références dans les textes médiévaux et l'importance de la voix dans la transmission; l'intervocalité est une "polyphonie perçue par les destinataires d'une poésie qui leur est communiquée quelles qu'en soient les modalités et le style performanciel - exclusivement par la voix » (1987: 161).

4 Cette dernière conception est particulièrement utile pour penser les références à Baudelaire et à sa poésie dans le rap. Les références ne sont pas seulement textuelles mais également performées à travers la voix, le flow, les samples, et même les clips comme le montreront nos exemples. Nous parlerons d'« intertextualité performée ", plutôt que d'intervocalité, puisque c'est bien le caractère littéraire des références qui est mis en avant dans les performances de manière plus ou moins ludique. La poésie de Baudelaire apparaît comme un point de repère culturel que les rappeurs s'approprient pour revendiquer leur légitimité artistique et poétique ou au contraire qu'ils rejettent afin de se démarquer du patrimoine littéraire français et des valeurs institutionnelles 
(Wallon 2012). Mais la présence de Baudelaire dans les morceaux de rap n'est pas qu'une question de légitimité poétique ou au contraire de revendication contrepoétique, elle fait avant tout partie d'un jeu sonore. Les exemples sélectionnés pour cet article montrent comment, en jouant et en déjouant la poésie de Baudelaire, les artistes de rap mettent en performance - en mouvance, pour utiliser un terme de Zumthor - le passé littéraire. Les exemples d'intertextualité performée, loin d'être exhaustifs, démontrent la diversité des références baudelairiennes et sont présentés selon une progression des références qui vont des plus explicites et complètes aux plus implicites et sommaires.

\section{Adaptations : parodie, sampling, intermédialité, paraphrase}

De nombreux rappeurs font explicitement référence à Baudelaire dans leurs textes, $\mathrm{y}$ compris des rappeurs connus du grand public tels que IAM dans "Sur les remparts " (2007), Orelsan dans «Jimmy Punchline» (2009), et Booba dans «Centurion» (2017). Cependant les artistes de hip-hop ne mettent pas réellement en musique les poèmes de Baudelaire, ils ne les rappent pas car l'existence même des rappeurs passe par une singularité de l'écriture, à la fois musicale et performative. Comme l'explique Julien Barret, l'écriture de rap est " un exercice de style " pour lequel la rime est encore plus importante qu'en poésie ou en chanson (2008: 22). Il est donc rare pour un ou une artiste de rapper un texte préexistant qui n'émerge pas de son esthétique propre. Les quatre catégories d'adaptation suivantes - la parodie, le sampling, l'intermédialité et la paraphrase - montrent qu'en transformant la poésie de Baudelaire les artistes font preuve de créativité en retravaillant les poèmes et en les associant à des performances singulières. Les références à Baudelaire ne sont pas simplement juxtaposées mais incorporées aux performances.

6 Les rares mises en musique rap des poèmes de Baudelaire sont généralement effectuées dans le cadre de spectacles ou de vidéos s'adressant aux élèves et étudiants dans une perspective de vulgarisation. Le spectacle suisse « Baudelaire Expérience » (2014) de DJ Eagle et Roccobelly a proposé une performance " psychédélique et hallucinatoire ${ }^{7}$ » des poèmes des Fleurs du mal et des Paradis artificiels. De même, l'humoriste Richard Sabak a adapté et mis en scène «À une passante » (2016) et «L'Albatros » (2017) dans deux clips de rap ${ }^{8}$. Dans ses adaptations de Baudelaire, l'humoriste parodie à la fois la poésie et le rap. La parodie musicale peut se définir comme une exagération de traits musicaux d'une composition, d'un genre, ou d'un musicien, à des fins comiques ou satiriques dans un morceau de musique (Tilmouth 2001). Dans les mises en rap de Sabak, le registre comique passe par la caricature des poèmes, par exemple les cris d'oiseau et la rime «A.L.B.A.T.R.O.S lance S.O.S!», mais également par la caricature des rappeurs. Les interjections et onomatopées sont exagérées dans les deux morceaux et la mise en scène d'une clique dans "À une passante" participe au ressort comique de ces parodies. L'humoriste ne ridiculise pas pour autant le rap et la poésie. Le respect de la prosodie poétique, notamment du e muet, et la justesse du placement vocal dans le style rap montrent que ces morceaux sont un hommage à la fois à la poésie et au rap plutôt qu'une dévalorisation des deux genres. Linda Hutcheon, qui a écrit une théorie de la parodie, explique que la parodie n'est pas faite pour ridiculiser mais plutôt pour apporter une distance critique qui passe par l'ironie $(2000: 6)^{9}$. Il y a en effet une 
distanciation ironique dans les musiques de Sabak puisqu'il ne s'agit pas seulement de parodier la poésie et le rap mais également de vulgariser la poésie de Baudelaire. Ces performances montrent que rapper les poèmes de Baudelaire ne peut se faire que par une distanciation explicite, qui passe par le cadre scolaire ou par l'humour. Ces mises en rap interrogent à la fois le rap et la poésie en tant que genres supposément sérieux à travers une confrontation entre hommage et légèreté.

7 Le sampling est une forme d'adaptation particulièrement prisée par le rap. Pour Isabelle Marc Martínez, l'idée de recyclage fait entièrement partie de l'esthétique du rap, elle définit le sampling comme une "utilisation des techniques de reproduction sonores, création collective, description et dénonciation du contexte politique et social contemporain » (2008: 35). Dans «Balaclava ${ }^{10} »(2017)$, l'artiste Koro\$hima utilise une récitation des sept premiers vers de «Spleen : j'ai plus de souvenirs que si j'avais mille ans » par Fabrice Luchini en introduction du morceau. Comme avec la parodie, ce sample introduit une distanciation entre le poème et le rap de Koro\$hima. Le poème de Baudelaire est ainsi mis en exergue par le sample, il est signalé comme élément externe $^{11}$. Les effets sonores ajoutés à la voix de Luchini renforcent l'idée d'étrangeté du poème. Une écoute plus attentive de l'extrait choisi montre cependant un lien thématique entre le poème et la performance de Koro\$hima. Le début du poème met en scène un poète accablé par son passé qui apparaît en même temps comme une figure hyperbolique dont le cerveau est « une pyramide, un immense caveau » ([1861] 1975 : 79). Au registre lyrique du poème répond l'égotrip de la performance du rappeur. De plus, l'intertextualité performée est particulièrement explicite dans ce morceau. En ayant pour thème le passé, le sample de «Spleen » s'auto-signale comme emprunt au patrimoine littéraire. Le fait que ce soit une récitation de Fabrice Luchini, dont la voix est immédiatement reconnaissable, souligne par ailleurs une mise en abîme de la performance. Mark Katz explique que les samples citent avant tout des performances qui représentent elles-mêmes des sons particuliers: "Le sampling digital offre la possibilité de ce que j'appellerais la citation performative : une citation qui recrée tous les détails de timbre et de temps qui évoquent et identifient un unique événement-son $[\ldots]^{12}$ » (2010: 149). Ici, il y a plusieurs événements-sons enchâssés les uns dans les autres - le poème de Baudelaire, la voix de Luchini, la performance de Koro\$hima - qui soulignent le caractère ludique des emprunts.

Une autre manière d'adapter explicitement la poésie de Baudelaire dans le rap est de recourir à l'intermédialité, c'est-à-dire d'entremêler la littérature, la musique et la peinture sur un même support. C'est ce qu'a fait Abd Al Malik avec son projet Le Jeune Noir à l'épée (2019), un livre album basé sur l'exposition «Le Modèle noir de Géricault à Matisse » (Musée d'Orsay, 2019). Le livre est constitué d'un récit poétique, de poèmes de Baudelaire, de peintures et de photographies, et est accompagné d'un album de neuf titres. Dix poèmes de Baudelaire sont insérés dans le livre (neuf poèmes de la section "Spleen et Idéal» des Fleurs du mal et un poème en prose), dont certains sont légèrement modifiés pour correspondre au message émancipateur que l'artiste veut transmettre. Par exemple le quatrain suivant est ajouté à trois reprises au refrain existant de "L'invitation au voyage » : «Regarde le monde est ainsi fait / Chacun veut juste avoir un avenir / Regarde le monde est ainsi fait / Chacun veut juste pouvoir s'en sortir » (Abd Al Malik 2019 : 74-75). L'artiste respecte la structure ternaire du poème et transforme l'idée de s'extraire du quotidien dans une perspective sociale contemporaine. Par ailleurs, le récit poétique fait référence au recueil Les Fleurs du mal plusieurs fois pour comparer les rappeurs aux poètes : «Et le rappeur qui est le Poète 
d'aujourd'hui, se confond avec le paradoxe, les incohérences, de l'époque qui font cohabiter le Ciel et l'Enfer, les ténèbres et la lumière» (2019: 151). Abd Al Malik met des majuscules aux mêmes mots que Baudelaire et transforme son style en une oralité qui lui est propre. En effet, les virgules imposent des pauses dans le récit et invitent à un tempo de lecture (silencieuse ou orale) lent. Les références à la poésie et au style de Baudelaire sont toujours signalées comme telles et permettent à l'artiste de revendiquer la filiation entre son rap et la poésie. Il y a là encore une distanciation avec les poèmes. Sur l'album, quatre morceaux contiennent des strophes du poème de Baudelaire «Bénédiction ». Abd Al Malik ne récite que deux ou trois strophes de ce long poème à la fin de chaque morceau et marque une pause avant la récitation accompagnée de bruits de fond signalant ainsi le poème comme un texte étranger. Le livre album d'Abd Al Malik est une performance d'intermédialité qui fait coexister rap, récit littéraire, poésie et peinture. D'après Werner Wolf, l'intermédialité est une relation identifiable entre modes d'expression dans le but de créer un objet artistique doté de significations (1999: 37). Parce qu'il y a une relation intermédiale dans la création d'Abd Al Malik, le rap et la poésie ne se confondent pas mais s'imbriquent et s'influencent mutuellement.

9 Le dernier exemple d'adaptation est une paraphrase du poème en prose « Le Chien et le Flacon » par le chanteur Lonepsi qui apparaît en introduction de son EP Sans dire adieu (2018). Le morceau se démarque des autres chansons de l'album par un style vocal articulé plus proche du slam que du rap et par le fait qu'il apporte un double métadiscours: une explication du texte de Baudelaire et une explication de la démarche artistique de Lonepsi. Avec cette paraphrase, l'artiste demande au public de faire un effort intellectuel pour apprécier ses chansons et de ne pas céder à la facilité de certaines chansons actuelles. Lonepsi utilise le poème de Baudelaire pour se positionner en artiste intellectuel et légitimer la supériorité de ses compositions qui procureront au public «des émotions importantes». Le chanteur invoque la valeur élitiste de Baudelaire telle qu'elle est représentée par le système scolaire. L'adaptation paraphrasée du poème fait d'ailleurs figure d'exposé scolaire avec une introduction, un développement et une conclusion. D'après Christian Béthune, l'une des particularités $\mathrm{du}$ rap francophone est justement de jouer avec le patrimoine littéraire à travers la culture scolaire (2003: 214). Ainsi, la performance musicale de Lonepsi, calme et mélancolique, s'inscrit dans une culture supposément légitime qui contraste avec la violence revendiquée de certains rappeurs.

Ces exemples variés d'adaptation opèrent une sorte d'ekphrasis musicale qui donne à entendre la poésie de Baudelaire. Cependant, les poèmes ne sont pas utilisés pour euxmêmes mais sont un moteur créatif. La poésie de Baudelaire est utilisée comme plateforme créative, elle ne peut être mise en musique rap car, par définition, le rap adapte et transforme plusieurs matériaux à la fois. Si les artistes signalent explicitement l'emprunt, ils n'hésitent pas à manipuler les poèmes pour créer une distance avec le texte original. Cette manipulation montre que le canon littéraire n'est pas intouchable: "L'effet de collage postmoderne détruit, par ailleurs, le concept d'unité et de monumentalité de l'art. Les rappeurs ne conçoivent pas leurs créations comme des œuvres éternelles et immuables » (Marc Martínez 2008 : 38). 


\section{Allusions : démarcation, citation, urbanité}

11 Alors que l'adaptation est explicite, l'allusion n'est identifiable que si l'on connaît la poésie de Baudelaire. Pourtant, comme pour l'adaptation, l'un des moteurs de l'allusion semble être le pouvoir inspirant de la référence baudelairienne. Le texte référencé, l'intertexte, est une force productive :

le concept d'intertexte est ce qui apporte à la théorie du texte le volume de la socialité : c'est tout le langage, antérieur et contemporain, qui vient au texte, non selon la voie d'une filiation repérable, d'une imitation volontaire, mais selon celle d'une dissémination - image qui assure au texte le statut, non d'une reproduction, mais d'une productivité. (Barthes 1973)

L'allusion dans le rap, qu'elle corresponde à une référence thématique ou à l'inclusion de vers, participe à la dissémination d'un mythe baudelairien, que ce soit pour l'encenser ou le désacraliser.

L'allusion au seul patronyme, ou au titre Les Fleurs du Mal, est souvent utilisée comme point de repère culturel embrassé ou rejeté par les artistes. Elle est révélatrice des tensions de hiérarchie entre le rap et la poésie, mais aussi entre les différents genres de rap. Baudelaire est une figure culturelle exploitée par les artistes de rap mais aussi par la critique de rap; François Dubet déclarait en 1996: "NTM n'est pas Baudelaire » (Libération, 18 novembre 1996), alors que Jonathan Franzen écrivait que les Baudelaire d'aujourd'hui sont les artistes de hip-hop ("Voices of the Wilderness ", The Guardian, 2002). Lorsque les allusions sont plus développées, les rappeurs se rattachent au patrimoine littéraire et célèbrent Baudelaire. Par exemple, dans « Rose noire » (2015) Georgio fait allusion au recueil de Baudelaire dans un morceau sur la rupture amoureuse pour installer une métaphore filée de la fleur: «Les fleurs du mal ne poussent pas qu'dans les poèmes de Baudelaire / C'est au bouquet final que tu dis que l'amour c'est la guerre ». De même dans " Hymne à la rime » (2012) le rappeur L'Indis reconnaît que Baudelaire influence ses compositions autant qu'Eminem : « Ma rime est gratis, me guérit d'tout c'qui m'égratigne / Elle a du respect pour Hugo, Baudelaire, Eminem et Rakim ». En revanche, de nombreux artistes de rap rejettent et insultent Baudelaire pour mieux revendiquer la légitimité du rap, comme Sadek qui qualifie sa composition de « poème » dans « Banlieue » (2015) : «Vive Le Rat Luciano rien à foutre de Baudelaire/Ouais ouais igo, j'préfère la plume d'Oxmo/Que les pièces de Molière / J'écris ce poème pour le ghetto ". D'autres artistes remettent en question la légitimité canonique de Baudelaire afin de souligner la domination coloniale. Dans « Le Flow du mal » (2012), RAD (la Radance) se qualifie de «Black Baudelaire » et Limsa, qui a par ailleurs sorti un EP intitulé Les Fleurs de Limsa, se définit comme un "Charles Baudelaire rebeu» («Freestyle Tohu Bohu», 2014). D'autres artistes préfèrent se référer à Aimé Césaire ou à Frantz Fanon dans leurs textes, comme la rappeuse Casey ou le rappeur Youssoupha ${ }^{13}$. De plus, hormis le duo de rappeuses Orties qui a choisi son nom en référence à Baudelaire, nous n'avons pas trouvé de rappeuses citant Baudelaire, comme si une certaine domination patriarcale était rejetée à travers le poète. De même, nous n'avons pas trouvé de références aux poétesses ${ }^{14}$.

13 L'allusion baudelairienne passe également par un placement subtil de citations chez certains artistes qui se revendiquent comme héritiers de Baudelaire en excluant en même temps une partie du public. Dans les exemples d'adaptation cités plus haut, la mention explicite à Baudelaire et la distanciation créée par les différents formats signalent l'emprunt et invitent, d'une certaine manière, tous les auditeurs à poursuivre 
la lecture de Baudelaire. Dans le cas de citations non signalées, les références incorporées mettent en place un jeu de restitution. Dans «Enivrez-vous 2 » (2017), Rochdi utilise quelques lignes du poème en prose «Enivrez-vous " pour son refrain : «Enivrez-vous mes amis / De vin, de poésie ou de vertu à votre guise / Mais enivrezvous mes amis / Enivrez-vous mes amis / La vie est si fragile, tôt ou tard il faudra partir». L'apostrophe "mes amis» montre que le rappeur s'adresse à un public restreint, celui qui peut comprendre la référence. Bien que Rochdi mentionne Baudelaire et Verlaine plus loin dans son morceau, la citation du poème en prose n'est pas identifiable si l'on ne connaît pas le poème. Le rappeur, qui a fait une thèse en philosophie de l'art, revendique la valeur savante et excluante de ses morceaux à travers une autre référence baudelairienne: "J'ai dansé avec les serpents enivrés, défoncés, alcoolisés à délirer / La poésie m'a transformé / J'ai plus le temps de rester auprès des ignorants, Dieu est grand». Par ailleurs, contrairement aux nombreux rappeurs qui convoquent la figure de Baudelaire pour le côté traditionnel et bienpensant, Rochdi fait allusion à l'image non-conformiste du poète car il souhaite luimême rester un artiste de l'ombre. Comme Rochdi, d'autres artistes font allusion à l'ivresse et à la drogue décrites par Baudelaire dans Les Paradis artificiels comme Dooz Kawa dans «Les fleurs poussent en décembre » (2018) ou Nadir qui rappe "J'ai trop fumé les fleurs du mal et en découle mon humeur buissonière » dans "Littérature " (2011).

Pour d'autres rappeurs, lorsque la citation est explicite, elle n'est pas incorporée dans les textes mais fait office de paratexte qui s'ajoute à la performance. C'est le cas du clip de « VLB» de Lino (2015) qui affiche à la toute fin la citation de Baudelaire «Tu m'as donné ta boue et j'en ai fait de l'or» extraite d'«Ébauche d'un épilogue pour la deuxième édition des Fleurs du mal ». Cette citation explicite devient une signature de la performance audiovisuelle et démontre que le rappeur revendique l'esthétique poétique de ses compositions; la "boue " peut être comprise comme la misère du quotidien changée en art mais également comme le passé littéraire rejeté et transformé.

Enfin, l'allusion peut se faire par rapprochement thématique, notamment dans les descriptions modernes de l'urbanité similaires dans la poésie de Baudelaire et dans certains textes de rap. Dans "Visages dans la foule » (2003) IAM décrit la foule de la cité comme « des âmes englouties "; le protagoniste perdu dans la foule est " un fantôme " et la mère qui vit dans les « tristes tours grises dans la banlieue d'Paris » est « un visage anonyme, un ange esseulé ». Ces images rappellent ce qu'observe le promeneur solitaire dans le poème en prose de Baudelaire «Les foules ». Le flâneur croise dans « la multitude, la solitude » de la ville " des âmes errantes qui cherchent un corps » ([1869] 1975 : 291). De plus, les descriptions de la rue et de la cité, souvent sombres, dans certains textes de rap font écho à la section "Tableaux parisiens» des Fleurs du Mal. Dans le poème « Les sept vieillards ", la ville est «fourmillante », la rue est «triste », et inondée d' "Un brouillard sale et jaune » (87). De manière similaire, « Rue de la peine » (2016) de Kery James décrit une "rue des oubliés », une "rue sale ", avec "des épines et des fleurs fanées que la rue emprisonne ». Chez Baudelaire, tout comme dans les exemples de rap précédents, l'urbanité est un espace de confrontation violent entre l'artiste et la multitude : « les poèmes en vers des "Tableaux parisiens" mettent l'accent moins sur le spectacle de la cité que sur la violence qu'elle exerce sur l'observateur luimême, flâneur, homme des foules » (Compagnon 2003 : 116). Au-delà des allusions baudelairiennes à l'urbanité moderne dans le rap, Baudelaire, comme d'autres artistes 
canoniques, appartient à l'imaginaire urbain en tant que toponyme. Les noms de rues, de collèges et lycées, et de quartiers portant le nom du poète confèrent une tout autre dimension au vocable "Baudelaire " qui influence aussi les références dans le rap. Plusieurs quartiers s'appellent Baudelaire (comme à Torcy ou à Saint-Denis), et comme l'a montré le film La Haine (1995) un immeuble de la cité de la Noé à Chanteloup-lesVignes revêt une grande mosaïque de Baudelaire ${ }^{15}$. Baudelaire est donc un monument, au sens propre comme au sens figuré, dans les textes de rap. Les allusions, plus ou moins subtiles, montrent que certains rappeurs revendiquent une filiation poétique baudelairienne quand d'autres créent une contre-poétique. Les artistes de rap jouent avec les tensions hiérarchiques entre rap et poésie pour mieux les écarter. Ils montrent aussi par la variété des performances, que le canon littéraire est artificiel, que la poésie de Baudelaire est malléable et mouvante, et que le rap lui-même est mouvant, multiforme, à la fois savant et populaire.

\section{Résonances : patronyme sonore et échos du passé chansonnier}

16 Le rap est avant tout une musique verbale, ou plutôt les raps sont des musiques car chaque artiste est différent, chaque performance est éphémère ${ }^{16}$. La composition d'un rap est autant sonore que visuelle, et les emprunts à la littérature sont avant tout des emprunts sonores. La poésie et le patronyme de Baudelaire « résonnent » dans le rap parce qu'ils «sonnent » tout court. Ce jeu de résonances peut se comprendre d'un point de vue synchronique et d'un point de vue diachronique.

Si les exemples précédents ont montré comment la poésie de Baudelaire peut s'imbriquer dans les performances de rap, il reste à souligner que la majorité des références à Baudelaire consiste à insérer le patronyme seul ou le syntagme « les fleurs du mal " sans développer la référence. Baudelaire est un topos des textes de rap mais aussi, et peut-être avant tout, un cliché sonore. Si le sampling recycle et insère des matériaux sonores, "Baudelaire» devient en soi matériau sonore, une image sonore comme définie par Mark Katz: «Peu importent les matériaux utilisés, le sampling fonctionne comme un puzzle : un son est coupé en morceaux qui sont ensuite recollés ensemble pour former une "image" digitale de ce $\operatorname{son}^{17} »(2010: 147)$. Ainsi, on trouve de nombreux titres rappelant le recueil de poèmes mais ne poussant pas plus loin l'allusion : «Les Flammes du Mal » (Passi, 1997), « 64 mesures de Spleen » (Jazzy Bazz, 2012), « Baudelaire » (DI\#SE, 2019). La résonance purement sonore se voit d'autant plus à travers la présence nominale du poète. Le patronyme "Baudelaire" devient luimême un vocable avec lequel les rappeurs jouent par les rimes. La rime la plus fréquente est sans doute "Baudelaire / bol d'air " comme dans «Krystal - Quand la mélodie m'emporte» (2003) de Rochdi: «J'ai une pensée pour monsieur Baudelaire / En savourant l'atmosphère, un verre d'eau, un bol d'air ». Dans une perspective linguistique, on peut dire que «Baudelaire » correspond plus au signifiant (à la forme sonore du mot) qu'au signifié (au concept de fond). En parlant du Signifyin(g) dans le hip-hop, qui correspond à l'idée que les musiques et les danses afro-américaines dialoguent entre elles, Justin A. Williams explique que pour que cette répétition du passé ait lieu, il faut que le signifiant soit mis en avant, lui donner de l'importance pour lui-même $(2015: 207)^{18}$. Baudelaire, dont la prononciation varie selon les accents et les flows des artistes, devient un écho, voire une interjection, qui résonne dans les 
performances de rap, ce qui pour Christian Béthune constitue la véritable performance : « Le flow confère à la phônê une dimension signifiante qui porte au-delà du code sémantique à l'intérieur duquel les mots figurent. C'est par le truchement du flow qu'un texte poétique devient poésie en acte » (2004:80).

La résonance baudelairienne s'opère également d'un point de vue diachronique. Les exemples précédents ont montré une intertextualité performée plus ou moins explicite et plus ou moins consciente qui dépasse les notions binaires de rap ou poésie, hommage ou rejet, oral ou écrit, art savant ou art populaire. Au contraire, ils témoignent d'une hétérogénéité qui participe à la dissémination du passé (plus ou moins proche) pour former un réseau d'influences. De telles binarités étaient en réalité déjà questionnées et dépassées à l'époque de Baudelaire. Comme l'explique Helen Abbott, il est difficile de considérer Baudelaire comme une figure emblématique d'un art savant puisque luimême a été influencé par l'art (supposément) populaire, notamment la chanson populaire :

Baudelaire ne crée pas une poésie cacophonique, bruyante, inspirée par la musique extérieure entendue dans la sphère publique. Mais il ne crée pas non plus une poésie raffinée, distinguée, inspirée par la musique du domaine privé entendue dans les salons littéraires et artistiques. Il exploite cependant ces deux mondes sonores afin de contester le statut même de la poésie en tant que forme artistique unique, redéfinissant ainsi un système de valeur poétique ${ }^{19}$. (Abbott $2017: 5$ )

Abbott rappelle également que le premier texte publié de Baudelaire était une chanson satirique co-écrite avec Gustave Le Vavasseur pour se moquer d'un autre chansonnier, Pierre de Béranger (2017: 4-5). Baudelaire a en quelque sorte participé à une querelle entre poésie et chanson en remettant même en doute la qualité de poète de Béranger (Brunel 2014 : 74). Baudelaire a cependant loué un autre chansonnier, Pierre Dupont, et a également emprunté à la chanson populaire, parfois sur le mode parodique, pour ses poèmes (Robb 1993 : 243). Anthony Pecqueux explique que «le rap procède de la forme chanson: soit cette résolution d'une équation entre des paroles proférées selon une vectorialité cinétique et un support mélodique (ou accompagnement, etc.)» $2007: 18)$. Entre la poésie de Baudelaire et le rap, il y a bien un réseau d'influences qui passe par la chanson. Dès la fin du xix siècle, les poèmes de Baudelaire ont été mis en musique classique, dans des mélodies ${ }^{20}$ (pièces vocales avec accompagnement au piano), mais également dans des chansons populaires. Il est plus difficile de retrouver les traces de chansons populaires puisqu'elles n'étaient pas toujours notées et étaient interprétées, entre autres, dans des cafés-concerts ou cabarets parisiens comme Le Chat Noir. Parmi les écrivains et musiciens qui fréquentaient Le Chat Noir se trouvait le poète Émile Goudeau qui a publié en 1885 un recueil de poèmes intitulé Fleurs du bitume: petits poèmes parisiens fortement influencé par Baudelaire ${ }^{21}$. Un siècle plus tard, la chanson de rap «Fleur de bitume" (Swift Guad, 2008) ou encore les phrases «Fleur du béton pousse quand ça n'chante plus (fleur du bitume)» du projet Facteur X (2002) et « dans la rue et le bitume ne poussent que les fleurs du mal» de Scred Connexion (2009), démontrent une continuité, sans être recherchée ni consciente, avec la poésie urbaine et la chanson du $\mathrm{XIX}^{\mathrm{e}}$ siècle. Cette continuité se voit également avec l'un des chansonniers les plus célèbres du début du $\mathrm{xx}^{\mathrm{e}}$ siècle: Aristide Bruant. Connu pour chanter en argot, ce dernier a publié un recueil de chansons intitulé Dans la rue (1889-1895) et plus tard un roman, Fleur de pavé (1925) 22. Également habitué du Chat Noir, il a fondé un autre cabaret, Le Mirliton, où il divertissait la clientèle en chantant et en l'insultant. Bien avant le début du rap, le lien entre la rue, l'argot, la violence 
verbale et la musique était déjà établi. Au milieu du $\mathrm{xx}^{\mathrm{e}}$ siècle, certains chanteurs comme Léo Ferré ont voulu se démarquer de la chanson commerciale en s'inscrivant dans une tradition intellectuelle dont la mise en musique de poèmes faisait partie (Hawkins [2000] 2017: 28). Léo Ferré a ainsi mis en musique une cinquantaine de poèmes de Baudelaire. Le chanteur a fait l'objet en 1962 du premier volume de la collection Poètes d'aujourd'hui de Pierre Seghers consacrée aux chansonniers. D'autres chanteurs ont également fait l'objet de volumes de cette collection (Jacques Brel, Barbara, Georges Brassens), érigeant ainsi un panthéon de la chanson française. Dans les années 1960, tout comme au XIX siècle, il y avait une distinction entre chanson " intellectuelle» et chanson populaire. Il y avait également un rapprochement entre poésie et chanson, comme il y a aujourd'hui une comparaison entre rap et poésie, ainsi que rap et chanson. Certains rappeurs d'aujourd'hui revendiquent faire (également) de la variété et s'associent volontiers à la tradition chansonnière. Sur son livre album Le Jeune Noir à l'épée, Abd Al Malik a collaboré avec Gérard Jouannest, pianiste et compositeur de chanson française ayant travaillé, entre autres, avec Jacques Brel. De la chanson populaire qui a influencé Baudelaire, aux mises en musique (populaires et classiques) de ses poèmes au XIX ${ }^{e}$ siècle, puis aux chansons françaises des années 1960, la poésie baudelairienne a parcouru un long chemin avant d'arriver dans le rap. Qu'ils en soient conscients ou non, les rappeurs et rappeuses se référant à Baudelaire participent de cette dissémination et de cette histoire de la musique française qu'a inspirée la poésie.

\section{Conclusion}

La référence à Baudelaire semble constante dans le rap depuis les premiers textes des années 1990 recensés sur Genius.com jusqu'à aujourd'hui. Si l'on note une légère augmentation des références baudelairiennes ces cinq dernières années, c'est parce qu'elles sont plus facilement identifiables grâce aux plateformes de streaming permettant aux artistes de rendre leurs morceaux accessibles mais aussi par un regain d'intérêt pour le poète lors du cent-cinquantenaire de sa mort en 2017 et du bicentenaire de sa naissance en 2021. Les performances variées de la poésie de Baudelaire dans le rap montrent qu'il existe un continuum entre mélodie, chanson, poésie et rap et que ces formes artistiques coexistent et s'influencent sans qu'il n'y ait de rapport hiérarchique. Il n'est pas question de filiation mais de transformation. Le rap, comme la poésie et la chanson, trouvent dans les matériaux du passé une plateforme créative. Le rap assume ses emprunts, que ce soit en cadrant explicitement les adaptations (par la parodie, le sampling, l'intermédialité, la paraphrase), en utilisant l'allusion pour un groupe restreint d'auditeurs (par la citation, la démarcation, la thématique urbaine), ou en utilisant les résonances de la sonorité du patronyme Baudelaire et du passé musical. Il y a donc l'idée d'une création collective qui démythifie l'image du seul génie créateur (Abbott 2019).

21 Le rap offre diverses mises en performance de la poésie et de la figure de Baudelaire qui démontrent que c'est un genre hybride mais également un genre pluriel. Chaque performance ne mentionne pas Baudelaire pour les mêmes raisons et, pourrait-on dire, ne mentionne pas le même Baudelaire. Que les artistes souhaitent s'inscrire dans une tradition littéraire ou rejeter cette tradition pour revendiquer une autre légitimité, ils participent tous à rejouer et disséminer le passé (Ghio 2016 : 244). Même les artistes qui 
semblent confondre Baudelaire avec un autre poète - dans une battle avec Chacalcolik, Yes McCann s'exclame "Y s'met du rouge à lèvres pis une perruque de Baudelaire ", et Joker veut «voguer sur le bateau ivre de Baudelaire»- mettent en performance, en mouvance, un passé littéraire.

Comme l'a montré le Baudelaire Song Project, la figure baudelairienne compte autant que la poésie dans l'inspiration des adaptations de certains genres musicaux ${ }^{23}$. Les artistes de rap sont par exemple particulièrement attirés par l'image du poète drogué et par l'image du poète condamné. Lors de la première publication des Fleurs du mal en 1857, Baudelaire a été condamné à payer une amende pour délit d'outrage à la morale publique et aux bonnes mœurs et à retirer six de ses poèmes. En 1949, le procès a été révoqué et la première version des Fleurs du mal a été réhabilitée, montrant ainsi que la conscience publique change et que ce qui est considéré comme offensant un jour ne le sera pas toujours. Certains avocats invoquent régulièrement aujourd'hui «la jurisprudence Baudelaire ${ }^{24}$ » pour défendre des rappeurs accusés d'outrage ou de diffamation. La mythologie baudelairienne continue ainsi de se créer à travers le rap, démontrant que Baudelaire parle à tous. Les adaptations de Baudelaire dans le rap, en conjonction avec les mises en musique dans d'autres styles, attestent d'une résonance collective depuis le xIX ${ }^{e}$ siècle jusqu'à aujourd'hui. C'est là que se trouve le pouvoir évocateur de Baudelaire qui a lui-même rejoué et déjoué le passé.

\section{BIBLIOGRAPHIE}

Abbott, Helen, 2017, Baudelaire in Song: 1880-1930, Oxford, Oxford University Press.

Abbott, Helen, 2019, « Collective Creativity: Negotiating Canonicity Through Adaptations of Baudelaire ", Modern Languages Open, $\mathrm{n}^{0}$ 1, p. 16, [En ligne], http://doi.org/10.3828/mlo.v0i0.229, consulté le 9 juillet 2021.

Abbott, Helen et Ardrey, Caroline, 2018, «"Mon gosier de métal parle toutes les langues": Translations and Transformations of Baudelaire in Black Metal Music ", L'Esprit Créateur, vol. 58, $n^{\circ}$ 1, p. 130-143, [En ligne], https://doi.org/10.1353/esp.2018.0010, consulté le 9 juillet 2021.

Abd Al Malik, 2019, Le Jeune Noir à l'épée, Paris, Présence Africaine, Flammarion, Musée d'Orsay, Musée de l'Orangerie.

Ardrey, Caroline, Abbott, Helen et Dubiau, Mylène, 2018, « Entre musique et lettres : vers une méthodologie numérique pour l'analyse de la mise en musique des poésies de Charles Baudelaire », Digital Humanities Quarterly, vol. 12, $\mathrm{n}^{\circ}$ 1, [En ligne], http:// www.digitalhumanities.org/dhq/vol/12/1/000364/000364.html, consulté le 9 juillet 2021. Barret, Julien, 2008, Le Rap ou l'artisanat de la rime. Stylistique de l'egotrip, Paris, L'Harmattan. Barthes, Roland, 1973, « Texte, théorie du », Encyclopcedia Universalis, [En ligne], http:// www.universalis.fr/encyclopedie/theorie-du-texte/, consulté le 9 juillet 2021.

Baudelaire, Charles, [1861] 1975, Euvres Complètes, vol. 1, Claude Pichois, Paris, Gallimard. Béthune, Christian, 2003, Le Rap, une esthétique hors la loi, Paris, Autrement. 
Béthune, Christian, 2004, Pour une esthétique du Rap, Paris, Klincksieck.

Brinker, Virginie, 2018, « Actualité de la pensée de Frantz Fanon dans le rap de Casey », Mouvements, vol. 96, $\mathrm{n}^{\circ}$ 4, p. 36-42, [En ligne], https://doi.org/10.3917/mouv.096.0036, consulté le 9 juillet 2021.

Brunel, Pierre, 2014, « Baudelaire et la musique : moderne ou antimoderne », L'Année Baudelaire, n $18-19$, p. 69-88, [En ligne], https://www.jstor.org/stable/45073924.

Compagnon, Antoine, 2003, Baudelaire devant l'innombrable, Paris, Presses de l'Université de ParisSorbonne.

Compagnon, Antoine, 2014, Baudelaire l'irréductible, Paris, Flammarion.

Genette, Gérard, 1982, Palimpsestes, Paris, Seuil, coll. « Poétique ».

Ghio, Bettina, 2016, Sans fautes de frappe. Rap et littérature, Marseille, Le Mot et Le Reste.

Ghio, Bettina, 2020, Pas là pour plaire! Portraits de rappeuses, Marseille, Le Mot et Le Reste.

Hannoosh, Michele, 1989, Parody and Decadence: Laforgue's Moralités légendaires, Columbus, Ohio State University Press.

Hawkins, Peter, [2000] 2017, Chanson. The French Singer-Songwriter from Aristide Bruant to the Present Day, Londres, Routlegde.

Hutcheon, Linda, 2000, A Theory of Parody: the Teachings of Twentieth-century Art Forms, Chicago, University of Illinois Press.

Katz, Mark, 2010, Capturing Sound: How Technology Has Changed Music, Berkeley, University of California Press.

Kristeva, Julia, 1969, Sémiotikè. Recherches pour une sémanalyse, Paris, Seuil.

Marc Martínez, Isabelle, 2008, Le Rap français : esthétique et poétique des textes (1990-1995), Bern, Peter Lang.

Pecqueux, Anthony, 2007, Voix du rap. Essai de sociologie de l'action musicale, Paris, L'Harmattan.

Robb, Graham, 1993, La Poésie de Baudelaire et la poésie française, 1838-1852, Paris, Aubier.

Samoyault, Tiphaine, 2001, L'Intertextualité. Mémoire de la littérature, Paris, Nathan.

Sangsue, Daniel, 1994, La Parodie, Paris, Hachette.

Tilmouth, Michael, 2001, « Parody », Oxford Grove Music Online, [En ligne], https://doi.org/ 10.1093/gmo/9781561592630.article.20938, consulté le 9 juillet 2021.

Wallon, Rémi, 2012, « "Va te faire niquer toi et tes livres" : Time Bomb, le triomphe d'un rap français "bête et méchant" ", Revue critique de fixxion française contemporaine, $\mathrm{n}^{\circ} 5$, [En ligne], http:// www.revue-critique-de-fixxion-francaise-contemporaine.org/rcffc/article/view/fx05.12, consulté le 9 juillet 2021.

Williams, Justin A., 2013, Rhymin' and Stealin': Musical Borrowing in Hip-Hop, Ann Arbor, University of Michigan Press.

Williams, Justin A., 2015, « Intertextuality, sampling, and copyright », dans J. A. Williams (dir.), The Cambridge Companion to Hip Hop Studies, Cambridge University Press, p. 206-220.

Wolf, Werner, 1999, The Musicalization of Fiction, A Study in the Theory and History of Intermediality, Amsterdam, Rodopi.

Zumthor, Paul, 1987, La Lettre et la Voix, De la « littérature » médiévale, Paris, Seuil. 


\section{Corpus}

Abd Al Malik, 2019, Le Jeune Noir à l'épée, Gibraltar Music Label.

Booba, 2017, « Centurion », Trône, Tallac Records, [En ligne], https://youtu.be/ao9gt6kgo6c, consulté le 9 juillet 2021.

DI\#SE, 2019, « Baudelaire », Parfum, Urban (PIAS), [En ligne], https://youtu.be/CwP6gceOgcI, consulté le 9 juillet 2021.

DJ Eagle, 2014, « Baudelaire Expérience », indépendant.

Dooz Kawa, 2018, «Les fleurs poussent en décembre ", L'indépendance en action 2, Larchiviste Records, [En ligne], https://youtu.be/bvi08nsymFU, consulté le 9 juillet 2021.

Facteur X, 2002, «Fleur du béton », Entretien avec un empire, Nouvelle donne Music, [En ligne], https://youtu.be/MCGK7lkgZ5M, consulté le 9 juillet 2021.

Georgio, 2015, « Rose Noire », Bleu noir, Musicast Distribution, [En ligne], https://youtu.be/ oJ8RgPd5Q_k, consulté le 9 juillet 2021.

IAM, 2003, « Visages dans la foule », Revoir un printemps, Hostile Records, [En ligne], https:// youtu.be/FwZskF41BYg, consulté le 9 juillet 2021.

IAM, 2007, « Sur les remparts », Saison 5, Polydor, https://youtu.be/-H4TKR_he_g, consulté le 9 juillet 2021.

Jazzy Bazz, 2012, « 64 mesures de Spleen », Sur la route du 3.14, indépendant, [En ligne], https:// youtu.be/RsHYz1YpQKI, consulté le 9 juillet 2021.

Kery James, 2016, « Rue de la peine », Mouhammad Alix, Musicast Distribution, [En ligne], https:// youtu.be/Elvulo8IBA8, consulté le 9 juillet 2021.

Koro\$hima, 2017, « Balaclava », indépendant.

Limsa, 2014, «Freestyle Tohu Bohu », indépendant, [En ligne], https://youtu.be/4uM_70SjukI, consulté le 9 juillet 2021.

L'Indis ft. Swift Guad, « Hymne à la rime », Mon Refuge, indépendant, [En ligne], https://youtu.be/ LaO6Jjs7n04, consulté le 9 juillet 2021.

Lino, 2015, «VLB », Requiem, Universal Music France, [En ligne], https://youtu.be/feH8G_Z6KOA, consulté le 9 juillet 2021.

Lonepsi, 2018, « Le Chien et le Flacon », Sans dire adieu, EP indépendant, https://youtu.be/ 4vfmP2R-cvU, consulté le 9 juillet 2021.

Lucio Bukowski ft. Nadir, 2011, «Littérature », Lucio Milkowski 2, indépendant, [En ligne], https:// youtu.be/r1RSUyc_7LE, consulté le 9 juillet 2021.

Orelsan, 2009, « Jimmy Punchline », Perdu d'avance, Wagram Music, [En ligne], https://youtu.be/ KuNUt43BVK0, consulté le 9 juillet 2021.

Passi, 1997, « Les Flammes du mal », Les Tentations, V2, [En ligne], https://youtu.be/3QGjx4a_Nfo, consulté le 9 juillet 2021.

Rad (La Radance), 2012, « Le flow du mal », Contre le peuple, indépendant.

Rochdi, 2017, « Enivrez-vous 2 », indépendant, [En ligne], https://youtu.be/KiQzfHv4Oos, consulté le 9 juillet 2021. 
Sabak, Richard, 2016, « À une Passante », indépendant.

Sabak, Richard, 2017, « L’Albatros », indépendant.

Scred Connexion, 2009, «Épingle du jeu », Ni vu...ni connu, Menace Records, [En ligne], https:// youtu.be/mohED5yENx8, consulté le 9 juillet 2021.

Sadek, 2015, «Banlieue », Johnny Niuum ne meurt jamais, Musicast Distribution, [En ligne], https:// youtu.be/eKwKInmEmDQ, consulté le 9 juillet 2021.

Swift Guad, 2008, « Fleur de bitume », Hécatombe, Horizone Prod, [En ligne], https://youtu.be/ 9Jaj9Z8tOVc, consulté le 9 juillet 2021.

\section{NOTES}

1. Comparaison effectuée en janvier 2021. Les chiffres sont changeants puisque Genius.com est une plateforme participative et que des paroles sont régulièrement ajoutées aussi bien pour les artistes professionnels qu'amateurs. Par ailleurs, de nombreuses paroles ne figurent pas encore sur le site, particulièrement pour les chansons issues d'albums du début des années 1990.

2. Projet de recherche (2015-2019) dirigé par Helen Abbott (University of Birmingham) en collaboration avec Mylène Dubiau (Université Toulouse-Jean Jaurès) et financé par l'Arts and Humanities Research Council.

3. Parmi les mises en musique recensées, plus de la moitié correspondent à de la musique classique, un quart peut être qualifié de chanson française, et un quart correspond aux autres styles (rock, metal, hip-hop, etc.). Voir www.baudelairesong.org/search, consulté le 9 juillet 2021.

4. Pour un explication détaillée du repérage et du traitement des données, voir Ardrey, Abbott et Dubiau (2018).

5. Par exemple Marie Jaëll (1846-1925), Rita Strohl (1865-1941) ou Marguerite Canal (1890-1978).

6. Les poèmes les plus mis en musique sont : «L'Invitation au voyage ", « La Mort des amants », « Recueillement », « Harmonie du soir », et « La Cloche fêlée ».

7. Voir https://www.youtube.com/watch?v=181RptYw888, consulté le 9 juillet 2021.

8. Ces clips ne sont plus disponibles sur YouTube mais des extraits de la musique peuvent être écoutés sur http://www.chartsinfrance.net/Richard-Sabak/albumssingles.html, consulté le 9 juillet 2021.

9. Sur la parodie, voir également Daniel Sangsue (1994) et Michele Hannoosh (1989).

10. Voir https://genius.com/Koro-rip-balaclava-lyrics, consulté le 9 juillet 2021.

11. Pour un autre exemple de poème samplé, voir Changstarr, "Fleurs du mal "악의 꽃" ", On the Road Mixtape vol. 2: Highdigger, indépendant, 2017. Le rappeur coréen utilise un sample de « Le Poison » au début de son morceau.

12. Nous traduisons. Citation originale : «Digital sampling therefore offers the possibility of what I would call performative quotation: quotation that recreates all the details of timbre and timing that evoke and identify a unique sound event [...]. »

13. Voir Virginie Brinker (2018) et Bettina Ghio (2020). 
14. Les romancières sont également peu citées; on trouve quelques références à Madame de Lafayette (à travers La Princesse de Clèves), à George Sand et à Marguerite Duras (par exemple citée dans « Thug Tropic » par Orties).

15. Quatre autres fresques de poètes revêtent les immeubles voisins : Arthur Rimbaud, Mallarmé, Gérard de Nerval et Paul Valéry. Le fait que ces fresques représentent des poètes (hommes) blancs dans une cité dont les habitants sont en majorité issus de l'immigration postcoloniale pose la question du sentiment d'aliénation que certains habitants peuvent ressentir. C'est aussi le cas pour de nombreux quartiers qui portent le nom d'écrivains blancs du xix ${ }^{e}$ siècle, souvent mentionnés par les rappeurs, telles la cité Balzac à Vitry dans les textes de Rohff ou la cité George Sand à Trappes dans les textes de La Fouine.

16. D'ailleurs, certains rappeurs mentionnés dans cet article ont retiré leurs chansons des plateformes de streaming.

17. Nous traduisons. Citation originale : «Regardless of the gear, on the simplest level sampling works like a jigsaw puzzle: a sound is cut up into pieces and then put back together to form a digitized "picture" of that sound. »

18. Nous traduisons. Citation originale: «to signify is to foreground the signifier, to give it importance for its own sake ».

19. Nous traduisons. Citation originale: «Baudelaire does not create cacophonous, noisy poetry inspired by the exterior music heard in public contexts, but neither does he create refined, gentle poetry inspired by the interior music encountered in the literary-artistic salons. Instead, he exploits both auditory worlds to challenge the very status of poetry as a stand-alone art form and to redefine a poetic value system. »

20. La première mise en musique documentée date de 1863 et est une composition sur «L'Invitation au voyage " par Jules Cressonnois.

21. Émile Goudeau, Fleurs du bitume : petits poèmes parisiens, Paris, Paul Ollendorf, 1885, [En ligne], https://gallica.bnf.fr/ark:/12148/bpt6k5543403v.texteImage, consulté le 9 juillet 2021. On retrouve de nombreux points communs entre Les Fleurs du mal et Fleurs du bitume. L'une des sections du recueil de Goudeau est intitulée "Cueillette sur l'asphalte» dont les poèmes sont consacrés aux femmes. Une autre section intitulée "Une saison de Spleen » traite de misère et de pauvreté.

22. Aristide Bruant, Dans la rue: chansons et monologues, Paris, Poulbot, 1889-1895, [En ligne], https://gallica.bnf.fr/ark:/12148/bpt6k719840/f1.image.texteImage, consulté le 9 juillet 2021; Aristide Bruant, Fleur de pavé, grand roman de pitié et d'amour, Paris, Taillandier, 1925, [En ligne], https://gallica.bnf.fr/ark:/12148/bpt6k1489305.image, consulté le 9 juillet 2021.

23. Voir Helen Abbott et Caroline Ardrey (2018).

24. Voir Dorian Chotard, "Un chanteur de rap poursuivi en diffamation depuis sept ans », Le Monde, 28 octobre 2009, [En ligne], https://www.lemonde.fr/societe/article/ 2009/10/28/quand-les-rappeurs-se-sentent-victimes-d-acharnement-

judiciaire_1259894_3224.html, consulté le 9 juillet 2021. 


\section{RÉSUMÉS}

Baudelaire est l'un des poètes qui a été le plus mis en musique. Cependant certaines adaptations de sa poésie sont trop éloignées du texte original pour être considérées comme mises en musique ; c'est le cas pour de nombreux morceaux de rap qui citent, samplent, ou paraphrasent des vers de Baudelaire. Cet article propose d'étudier les représentations et performances de la poésie de Baudelaire dans le rap français dans une perspective culturelle, textuelle et musicale. Le poète apparaît comme un point de repère culturel que les rappeurs s'approprient pour revendiquer leur légitimité artistique et poétique ou qu'ils rejettent au contraire afin de se démarquer du patrimoine littéraire français et des valeurs institutionnelles. L'intertextualité performée des poèmes de Baudelaire dans le rap montre cependant un dépassement des binarités rap et poésie, art populaire et art savant. Il se crée plutôt un riche réseau d'influences, qui est lié à la culture urbaine et qui repose sur un jeu de sonorités, entre la poésie de Baudelaire et le rap, en passant par la mélodie et la chanson françaises.

Baudelaire is amongst the French poets who have most often been set to music. However, some adaptations of his poetry are too loose to be considered as song settings. It is the case for numerous rap songs that cite, sample, or reword Baudelaire's poems. This article examines representations and performances of Baudelaire's poetry through a cultural, textual, and musical perspective. Baudelaire appears to be a benchmark that rap artists celebrate to legitimise their work or that they reject to mark a distance with the French literary canon and related institutional values. The performed intertextuality at stake shows however a revocation of traditional oppositions between rap and poetry, highbrow art and popular art. Instead, a rich network linked to urban culture and the exploitation of sonic connections is created between poetry, mélodie, chanson, and rap.

\section{INDEX}

Keywords : Baudelaire (Charles), hip hop, rap, adaptation, intertextuality, urbanity, song

Mots-clés : Baudelaire (Charles), rap, hip-hop, adaptation, intertextualité, urbanité, chanson

\section{AUTEUR}

NINA ROLLAND

University of Birmingham 